Review

\title{
Redox modulation of cellular signaling and metabolism through reversible oxidation of methionine sensors in calcium regulatory proteins
}

\author{
Diana J. Bigelow, Thomas C. Squier* \\ Cell Biology and Biochemistry Group; Biological Sciences Division; Fundamental Sciences Directorate, \\ Pacific Northwest National Laboratory, P. O. Box 999, Richland, WA 99352, United States
}

Received 12 August 2004; received in revised form 9 September 2004; accepted 13 September 2004 Available online 25 September 2004

\begin{abstract}
Adaptive responses associated with environmental stressors are critical to cell survival. Under conditions when cellular redox and antioxidant defenses are overwhelmed, the selective oxidation of critical methionines within selected protein sensors functions to downregulate energy metabolism and the further generation of reactive oxygen species (ROS). Mechanistically, these functional changes within protein sensors take advantage of the helix-breaking character of methionine sulfoxide. The sensitivity of several calcium regulatory proteins to oxidative modification provides cellular sensors that link oxidative stress to cellular response and recovery. Calmodulin $(\mathrm{CaM})$ is one such critical calcium regulatory protein, which is functionally sensitive to methionine oxidation. Helix destabilization resulting from the oxidation of either $\mathrm{Met}^{144}$ or $\mathrm{Met}^{145}$ results in the nonproductive association between CaM and target proteins. The ability of oxidized CaM to stabilize its target proteins in an inhibited state with an affinity similar to that of native (unoxidized) $\mathrm{CaM}$ permits this central regulatory protein to function as a cellular rheostat that down-regulates energy metabolism in response to oxidative stress. Likewise, oxidation of a methionine within a critical switch region of the regulatory protein phospholamban is expected to destabilize the phosphorylation-dependent helix formation necessary for the release of enzyme inhibition, resulting in a down-regulation of the Ca-ATPase in response to $\beta$-adrenergic signaling in the heart. We suggest that under acute conditions, such as inflammation or ischemia, these types of mechanisms ensure minimal nonspecific cellular damage, allowing for rapid restoration of cellular function through repair of oxidized methionines by methionine sulfoxide reductases and degradation pathways after restoration of normal cellular redox conditions.
\end{abstract}

(C) 2004 Published by Elsevier B.V.

Keywords: Methionine oxidation; Calmodulin; Signal transduction; Calcium regulation; Methionine sulfoxide reductase; Cellular metabolism

\section{Linkages between oxidative stress and energy metabolism: identification of control points}

The identification of oxidatively sensitive proteins that modulate energy utilization and the associated generation of reactive oxygen species (ROS) are critical to understanding how cells respond to oxidative stress. In this latter respect, oxidant-induced functional losses of the calcium regulatory proteins calmodulin $(\mathrm{CaM})$ and the sarcoplasmic/endoplasmic reticulum Ca-ATPase (SERCA) contribute to the downregulation of cellular metabolism and ATP utilization and

\footnotetext{
* Corresponding author. Tel.: +1 509375 2121; fax: +1 5093721632

E-mail address: thomas.squier@pnl.gov (T.C. Squier).
}

the associated generation of ROS associated with replenishing intracellular ATP through oxidative phosphorylation [1]. Reductions in the rate of ROS generation, in turn, will minimize protein oxidation and facilitate intracellular repair and degradative systems that function to eliminate damaged and partially unfolded proteins (Fig. 1). Age-dependent increases in the rate of ROS generation or declines in cellular repair or degradation mechanisms will increase the oxidative load on the cell, resulting in corresponding increases in the concentrations of oxidized proteins and the associated formation of protein aggregates, which have been extensively documented in aged tissues. These results suggest that the general mechanisms underlying oxidantdependent changes in cell function are likely to be relevant 


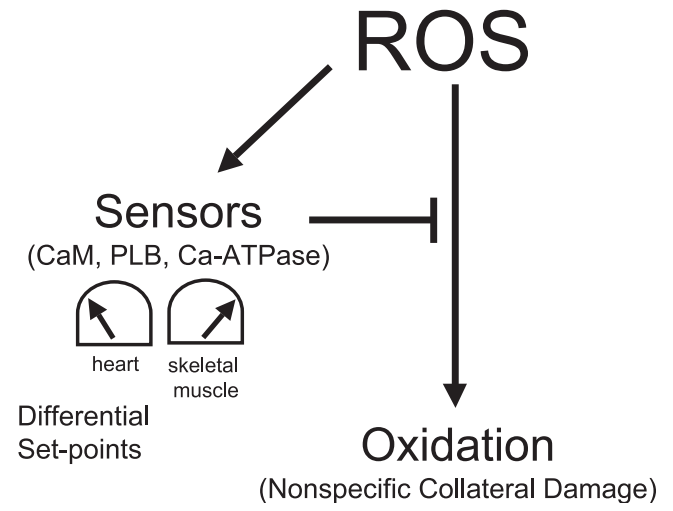

Fig. 1. Critical sensors of ROS determine cellular sensitivity to ROS. The functional sensitivity of key proteins to oxidative damage serves as a detector to balance metabolism, where there is a trade-off between optimal cellular function and the minimization of cellular damage. Oxidative modification of functionally sensitive sites on proteins that modulate calcium signaling and energy utilization will down-regulate ATP consumption through respiratory control mechanisms and minimize the generation of ROS and the associated nonspecific oxidative damage to cellular proteins. For example, helix destabilization in calmodulin (CaM) and phospholamban following methionine oxidation is proposed to stabilize the inhibited state of the Ca-ATPase. Likewise, direct nitration of tyrosines in the Ca-ATPase functions to decrease function. Differential setpoints of different organs are determined by the sensitivities of key sensors to oxidative and nitrative modification, which is determined by the reactivity of sensitive sites on regulatory proteins to particular ROS, endogenous antioxidant levels, protein repair, and rates of protein degradation. Thus, some systems (e.g., heart) have sensitive setpoints, which function to minimize nonspecific collateral damage, while others with greater regenerative capacities (e.g., skeletal muscle) tolerate larger amounts of cellular damage, permitting high levels of cellular function under conditions of oxidative stress.

to diverse pathologies, and that generalities are possible from a consideration of the aging process.

\section{Aging, metabolic rate, and adaptive cellular responses}

Biological aging represents a chronic oxidative stress that initiates a series of adaptive cellular responses. Understanding these control mechanisms, which permit optimal cell function and the management of oxidative stress, is critical to an appreciation of cellular stress responses. In this respect, caloric restriction results in an enhanced life span that correlates with the differential expression of critical proteins involved in energy metabolism, stress responses, and calcium regulation [2-4]. These results suggest tradeoffs during the aging process between the energetic costs associated with the maintenance of intracellular calcium gradients necessary for cellular signaling, oxidative damage associated with respiration, and physiological responses associated with stress responses. Consistent with this latter concept, $30-40 \%$ increases in life span and corresponding decreases in age-related diseases are associated with caloric restriction in mammals that correlate with a reduction in basal energy metabolism [3,5]. Thus, a decrease in metabolic rate and the associated generation of ROS appears to be critical to the maintenance of cell function during aging.

\section{Calcium regulatory proteins and linkages to energy metabolism}

Calcium functions as a critical second messenger in mediating fast intracellular responses in all eukaryotic tissues through the activation of $\mathrm{CaM}$ and other signaling proteins to coordinate cell function with energy metabolism. In addition, calcium mediates adaptive responses through the direct modulation of numerous transcription factors, including Nrf2, NFAT, and NF- $\kappa$ B family members c-Rel and RelA [6-9]. The sequence of events that modulates cell function involves the feedback modulation of the calcium signal through $\mathrm{CaM}$ binding to target proteins (Fig. 2). The cycle of calcium release and resequestration, i.e., the calcium transient, occurs in the following sequence: calcium influx into the cytosol occurs largely through calcium release channels in the intracellular compartment of the endo/sarcoplasmic reticulum or the plasma membrane. Finally, the calcium signal is reset by the rate-limiting resequestration of calcium ions back into the SR/ER lumen by the SERCA or by efflux through the plasma membrane. In all cases, CaM functions as a key sensor of the calcium signal to modulate cellular metabolism through the reversible binding to over 50 different target proteins, including calcium pumps and channels [10]. Thus, CaM functions to interpret the calcium signal to function as a feedback inhibitor of the calcium channels and a feed-forward activator of the calcium pumps to modulate the calcium signal and rates of intracellular metabolism, which in turn affects the generation of ROS, including superoxide, hydrogen peroxide, and peroxynitrite.

Key linkages between calcium regulation, energy metabolism, and intracellular signaling can be illustrated in the case of the heart, where regulation of the calcium transient is primarily through the $\beta$-adrenergic cascade. The $\beta$-adrenergic signaling cascade provides for increased rates of relaxation and force of contraction. This regulation impinges upon the calcium transient at three major points, by phosphorylation of calcium channels, troponin I, and phospholamban, which results in calcium channel activation, enhanced force generation, and enhanced rates of calcium resequestration into the SR, respectively [11]. This rapid release and resequestration of calcium in the heart on a beat-to-beat basis requires large amounts of energy; the diminished amplitudes and elongated times associated with calcium transients in aged hearts represent an adaptive mechanism that has the effect of reducing the energy demands and associated production of ROS [11,12]. Decreasing energy demand on the aged myocardium takes on additional importance if there are impairments in the function of key components of oxidative metabolism, such as the $\alpha$-ketoglutarate dehydrogenase complex and aconi- 


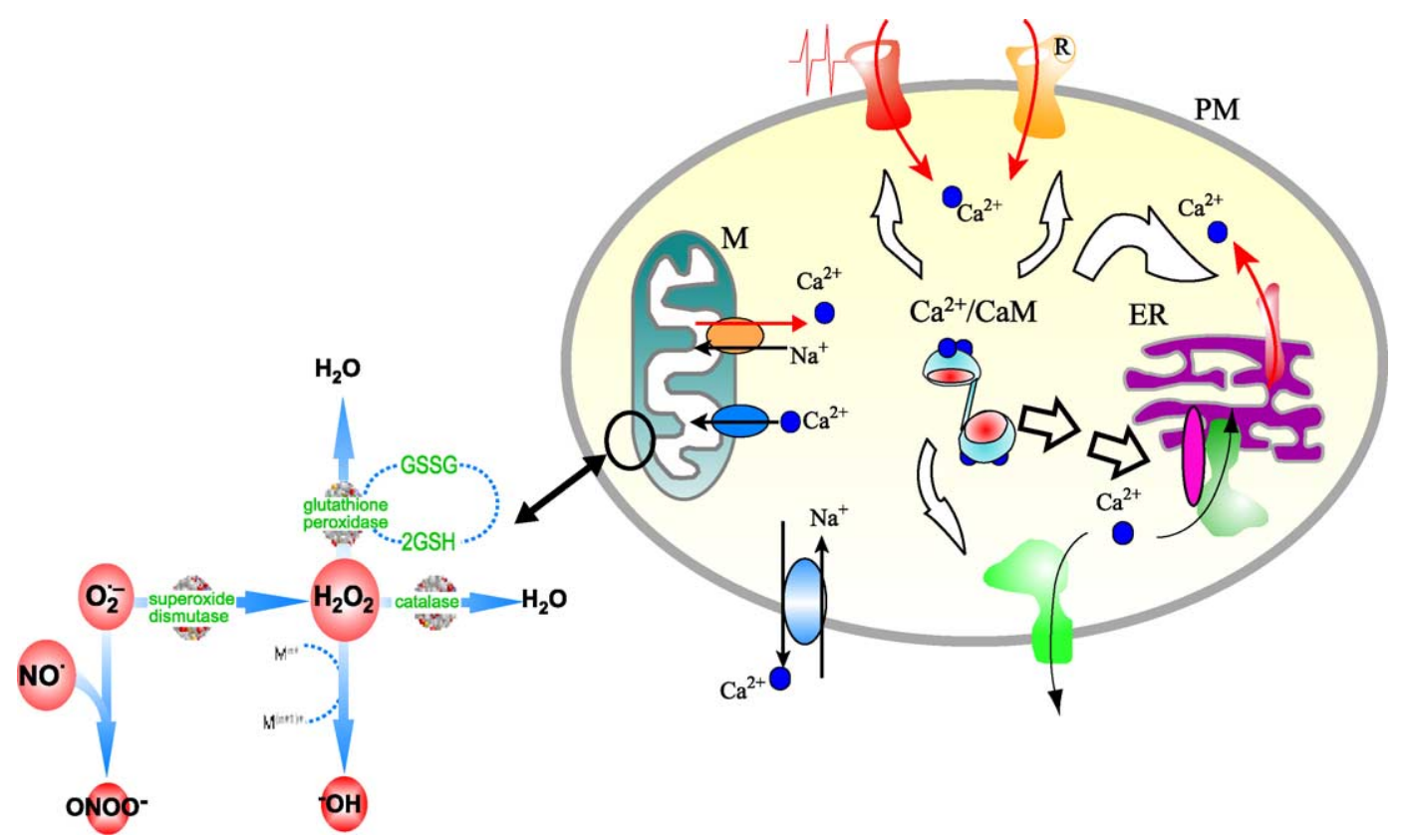

Fig. 2. Calcium transport systems and relationships to oxidative stress. $\mathrm{Ca}^{2+}$-activated CaM functions to enhance the activities of the SERCA and PMCA calcium pumps (green), which are respectively located in either the endoplasmic reticulum (ER) or plasma membrane (PM). Regulation is either through the phosphorylation of phospholamban (magenta), the protein that regulates SERCA, or direct binding to the autoinhibitory domain of the PMCA. Similarly, CaM associates with voltage- and ligand-gated calcium release channels and functions to inhibit calcium release at high intracellular calcium concentrations. Additional regulation of calcium involves sodium/calcium antiporters and calcium symporters in the mitochondria (M) and PM. Calcium entry into the mitochondria regulates dehydrogenases associated with the citric acid cycle and directly modulates energy production. Further regulation involves respiratory control mechanisms where ATP utilization through the maintenance of calcium gradients regulates flux through the electron transport chain and modulates the generation of ROS. Normal antioxidant defense mechanisms associated with the detoxification of ROS are highlighted, where superoxide $\left(\mathrm{O}_{2}^{--}\right)$produced during normal cellular conditions is dismutated to form hydrogen peroxide $\left(\mathrm{H}_{2} \mathrm{O}_{2}\right)$ by SOD. $\mathrm{H}_{2} \mathrm{O}_{2}$ is subsequently degraded to $\mathrm{H}_{2} \mathrm{O}$ by antioxidant enzymes such as catalase and glutathione peroxidases. $\mathrm{H}_{2} \mathrm{O}_{2}$ also can lead to the generation of the highly reactive hydroxyl radical ( $\left.\mathrm{OH}\right)$ through transition metal $\left(\mathrm{M}^{n+}\right)$ catalyzed reactions. Cellular messenger nitric oxide (NO) together with excessive $\mathrm{O}_{2}^{-}$can form highly reactive peroxynitrite $\left(\mathrm{ONOO}^{-}\right)$. Reduced and oxidized forms of glutathione are indicated as GSH and GSSG, respectively.

tase, which can result in the increased production of ROS [13]. Alternatively, down-regulation of flux through the electron transport chain resulting from decreases in energy metabolism or the oxidation of key transporters (e.g., the mitochondrial adenine nucleotide translocater) may compensate for a decreased functionality of electron transfer components $[1,14]$. By reducing the rate of ROS generation, normal cellular antioxidant defenses (e.g., superoxide dismutase and catalase), repair enzymes (e.g., MsrA and $\mathrm{MsrB}$ ), and degradative pathways (e.g., the proteasome) have the potential to maintain normal cellular function. Thus, it is likely that by decreasing cellular energy demands that normal function can be optimized by minimizing the accumulation of oxidized proteins and the associated formation of protein aggregates that can lead to protein aggregation and amyloid formation.

\section{Functional targets of oxidation during aging}

The selective and reversible oxidation of critical sites within proteins involved in signal transduction cascades would suggest a regulatory function analogous to previously recognized mechanisms involving protein phosphorylation.
It is, therefore, important to consider the extent of protein modifications that occurs under conditions of oxidative stress. Approximately one-half of intracellular proteins are oxidized in senescent animals, suggesting that during aging there is a nonselective oxidation of many cellular proteins [15]. However, in the majority of cases the oxidation of one or two amino acids has a minimal effect on protein function, altering neither the stability nor the function of the protein $[16,17]$. In contrast, some proteins are selectively oxidized at critical sites that regulate their function in a reversible manner. In this respect, the calcium regulatory protein $\mathrm{CaM}$, calcium pumps in both sarcoplasmic reticulum (SERCA) and the plasma membrane (PMCA), and calcium channels (e.g., the ryanodine receptor) are preferentially damaged by ROS $[1,18]$. In the case of CaM and SERCA, the structural and functional consequences associated with the oxidative modification of unique sites have been identified [19-26]. Thus, methionines in $\mathrm{CaM}$ are oxidized to their corresponding methionine sulfoxides, while the specific nitration of tyrosines in SERCA results in an age-related loss of function. Loss of Ca-ATPase function has broad implications, since the major utilization of ATP in excitable cells is for restoration of ion gradients, which are continually being dissipated through a variety of ion channels for the initiation 
of rapid physiological processes such as nerve conduction and muscle contraction. In muscle, the SERCA isoforms of the Ca-ATPase in the sarcoplasmic reticulum (SR) membrane are responsible for the rate-limiting removal of cytosolic calcium to the SR lumen that resets the 1000fold calcium gradient after each contractile event. In aging heart and skeletal muscle, the function of these ion transporters is diminished, resulting in slower calcium transients and prolonged relaxation times. Altered calcium transients may have longer-term effects, as well, related to calcium dependent transcriptional events.

\section{Calmodulin and mechanisms underlying inhibitory action of methionine oxidation}

$\mathrm{CaM}$ functions to interpret the calcium signal in all cells, and coordinates energy metabolism involving the mobilization of cellular energy reserves with gene transcriptional regulation through the differential activation of over 50 different target proteins (Fig. 2) [10]. Thus, modulation of the cellular concentration of CaM directly affects cellular function through the differential activation of low-affinity target proteins. This regulation follows from the observation that under all conditions, most $\mathrm{CaM}$ is bound to intracellular targets and that the concentration of binding targets exceeds the amount of CaM $[27,28]$. The fact that there are limiting amounts of cellular $\mathrm{CaM}$ implies that localized increases in cytosolic calcium redistribute bound $\mathrm{CaM}$ to activate different target enzymes. Depending on the time-duration and amplitude of the calcium signal, varying amounts of $\mathrm{CaM}$ are mobilized that can bind preferentially to target enzymes with differing CaM-binding affinities. Different classes of CaM-binding targets have been identified that bind calcium-activated $\mathrm{CaM}$ with differing affinities [29]. Thus, short-duration, low-amplitude calcium signals will mobilize small amounts of $\mathrm{CaM}$, which will preferentially bind to high-affinity targets, such as the plasma membrane CaATPase $\left(K_{\mathrm{d}}<10 \mathrm{nM}\right)$ (Fig. 3) [30]. In contrast, longduration, high-amplitude calcium signals will mobilize large amounts of $\mathrm{CaM}$ that will promote the association between $\mathrm{CaM}$ and a larger group of $\mathrm{CaM}$ binding partners, including those with relatively low affinities such as CaMdependent protein kinase II $\left(K_{\mathrm{d}}=30 \mathrm{nM}\right)$ and the epidermal growth factor $(\mathrm{EGF})$ receptor $\left(K_{\mathrm{d}}=400 \mathrm{nM}\right)[31,32]$.

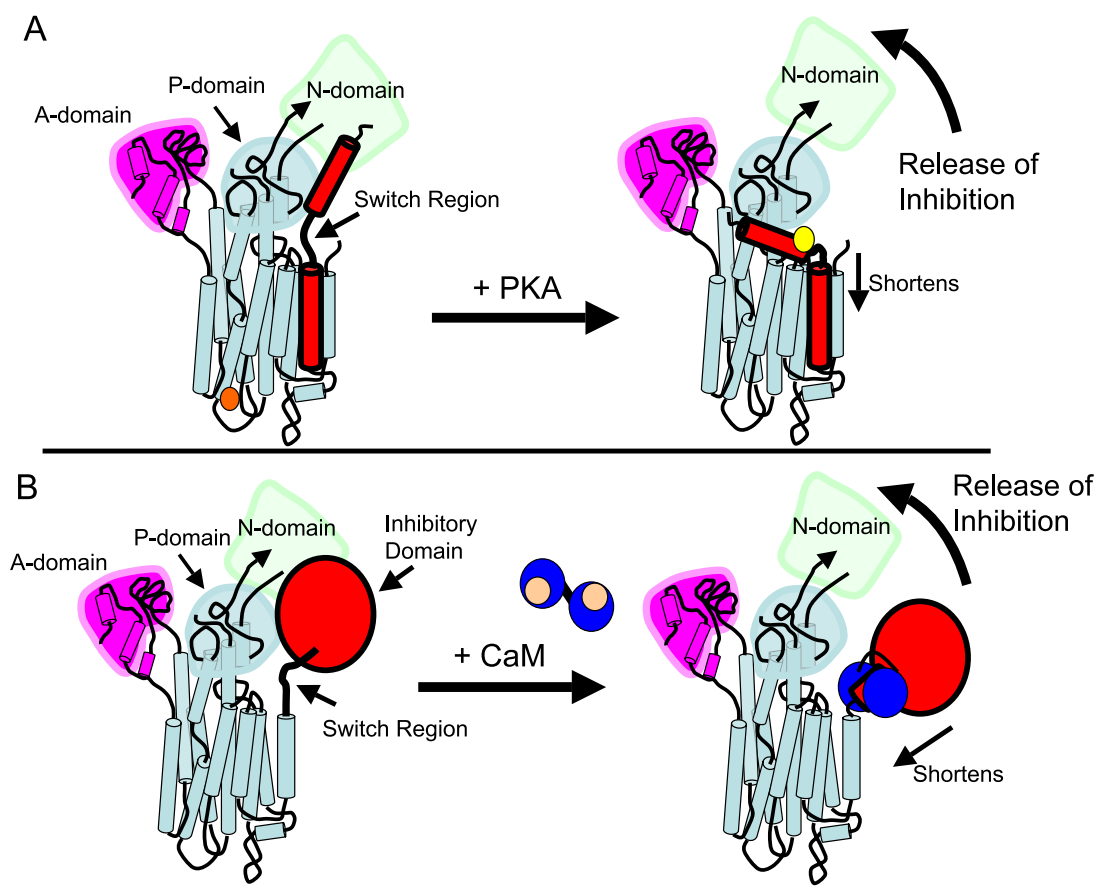

Fig. 3. Release of Ca-ATPase inhibition following stabilization of helix in switch region. Binding to the nucleotide binding domain (N) of the sarco/ endoplasmic reticulum Ca-ATPase (SERCA) underlying inhibitory interaction involves an extended switch region of either phospholamban (A) or the inhibitory domain (B) (shown in red), resulting in a reduction in catalytically important motions necessary for function of SERCA (A) or plasma membrane (B) Ca-ATPase. Stabilization of helical structure within a conformational switch region results in a shortening of the physical dimensions and the necessary release of the inhibitory interactions with the N-domain. Thus, in the example of SERCA (A), following phosphorylation of either Ser ${ }^{16}$ or Thr ${ }^{17}$ in phospholamban (yellow circle) by PKA or CaM-dependent protein kinase II (not shown), salt bridges are respectively formed with $\mathrm{Arg}^{13} \mathrm{or} \mathrm{Arg}^{14}$, inducing helical formation $[109,110]$. In the case of the plasma membrane Ca-ATPase (B), the inhibitory domain represents a $25-\mathrm{kDa}$ extension of the protein located at the C-terminal end of the protein which contains a CaM-binding sequence, and assumes a helical structure following CaM binding [113]. The SERCA and plasma membrane CaATPase are structurally homologous; each contains 10 transmembrane helices (aqua) and three distinct cytosolic domains, labeled N (light green; contains nucleotide binding site), P (aqua; contains site of covalent phosphorylation that triggers release of calcium), and A (magenta). High affinity calcium binding sites are located near the center of the bilayer, and critical calcium binding ligands are located on the helix containing Tyr ${ }^{294}$ and Tyr ${ }^{295}$ (orange), which are nitrated during biological aging [1]. Structural model of Ca-ATPase adapted from that of Toyoshima and Inesi [98]. 
In addition to the cellular regulation mediated by the transcriptional regulation of $\mathrm{CaM}$ expression, additional regulation occurs through reversible posttranslational modifications, which in the case of phosphorylation differentially modulates the binding affinity of CaM to different target proteins [33-35]. Likewise, the functional sensitivity of CaM to ROS provides a means to rapidly down-regulate cellular metabolism under conditions of oxidative stress [1]. In this respect, site-specific oxidative modifications to CaM may be mediated through the interaction with specific binding partners that include nitric oxide synthase (NOS) and superoxide dismutase (SOD); the latter is known to modulate calcineurin function in a redox-dependent process involving CaM [36,37].

Wild-type (unoxidized) CaM binds to the inhibitory domain of the plasma membrane Ca-ATPase, inducing helix formation within the CaM-binding sequence and releasing enzyme inhibition (Fig. 3B) [30,38]. In contrast, following oxidative modification of $\mathrm{Met}^{144}$ or $\mathrm{Met}^{145}$ in CaM, oxidized $\mathrm{CaM}\left(\mathrm{CaM}_{\mathrm{ox}}\right)$ binds to the autoinhibitory domain and stabilizes the inhibited enzyme state of the plasma membrane Ca-ATPase (Fig. 4) [19,23]. Likewise, other enzyme activities have been found to be modulated by oxidation of methionines in CaM, suggesting that there is a coordinate control of the function of different binding partners [25,26,39]. Since oxidation of methionines is reversible, and $\mathrm{CaM}_{\mathrm{ox}}$ is selectively degraded by the proteasome $[40,41]$, these results suggest that the site-

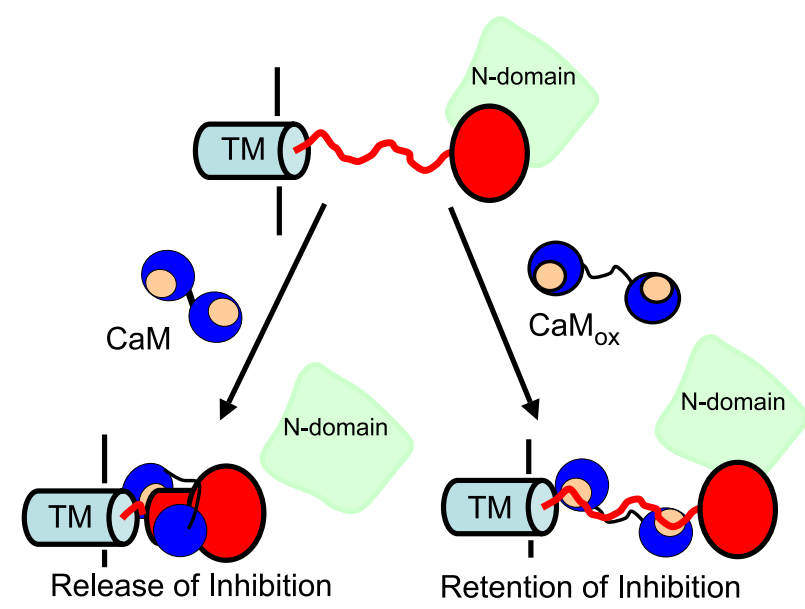

Fig. 4. Nonproductive binding of $\mathrm{CaM}_{\mathrm{ox}}$ following methionine oxidation. Helix stabilization within the CaM-binding sequence of the inhibitory domain of the plasma membrane Ca-ATPase (red) upon CaM binding functions as a conformational switch to release enzyme inhibition through association with the nucleotide $(\mathrm{N})$ binding domain of the Ca-ATPase. In contrast, the opposing domains of $\mathrm{CaM}_{\mathrm{ox}}$ are structurally uncoupled upon oxidation of $\mathrm{Met}^{144}$ or $\mathrm{Met}^{145}$, resulting in an altered binding interaction that does not promote helix formation and enzyme activation. Under this latter condition, the association is retained between the inhibitory domain and the N-domain of the Ca-ATPase, with an accompanying restriction in catalytically important domain motions associated with optimal transport activity. The transmembrane helix (TM) functions as a fixed point that defines the overall dimensions of the cytosolic portion of the inhibitory domain. specific modulation of $\mathrm{CaM}$ function by methionine oxidation is part of an adaptive cellular response to coordinate intracellular signaling, metabolism, and energy utilization.

\subsection{Oxidation of CaM during aging: differential sensitivities in brain and muscle}

Multiple methionines in CaM isolated from brains of Fisher 344 rats are oxidized to their corresponding sulfoxides; oxidation occurs progressively during aging and in senescent animals an average of two methionines are oxidized in each CaM [42]. Accumulation of oxidized CaM $\left(\mathrm{CaM}_{\mathrm{ox}}\right)$ occurs in spite of the relatively short half-life of $\mathrm{CaM}$, which is approximately $18 \mathrm{~h}$ in these animals [43]. Further, essentially all CaM is oxidized in skeletal muscle, while CaM isolated from the hearts of these same animals is not appreciably oxidized (unpublished observations). Thus, oxidation of $\mathrm{CaM}$ is not a biomarker of aging, as the accumulation of oxidized CaM does not occur in all tissues [44]. Rather, accumulation of $\mathrm{CaM}_{\mathrm{ox}}$ is the result of a combination of low antioxidant capacities, enhanced rates of ROS generation, and diminished repair or degradation systems in some tissues (i.e., skeletal muscle and brain) in this animal model.

The observation that methionines in $\mathrm{CaM}$ are selective targets under conditions of oxidative stress provides an important clue to adaptive cellular responses that might be missed normally. Furthermore, irrespective of whether $\mathrm{CaM}_{\text {ox }}$ is isolated from senescent tissue or oxidized under in vitro conditions, $\mathrm{CaM}_{\mathrm{ox}}$ stabilizes target proteins (e.g., the plasma membrane $\mathrm{Ca}$-ATPase) in an inhibited state, thereby diminishing ATP utilization under conditions of oxidative stress $[19,42]$. These results indicate that under conditions when ROS overwhelm antioxidant defenses, CaM is oxidized selectively and will down-regulate energy metabolism. Thus, methionine oxidation appears to be a primary signal that may play an important regulatory role is coordinating cellular signaling, energy metabolism, and calcium regulation. The fact that there are decreasing amounts of methionine oxidation in $\mathrm{CaM}$ isolated respectively from fast-twitch skeletal muscle, brain, and heart indicate differences in sensitivity of $\mathrm{CaM}$ to oxidation that reflect tissue-dependent differences in metabolism and antioxidant defenses (Fig. 1).

\subsection{Functional sensitivity determined by rate of oxidation of $\mathrm{Met}^{144}$ or $\mathrm{Met}^{145}$}

Oxidation of $\mathrm{Met}^{144}$ and $\mathrm{Met}^{145}$ near the carboxylterminus of CaM induces a nonproductive binding interaction that stabilizes the inhibited state of the plasma membrane Ca-ATPase (Fig. 5) [19,20,23,45]. Oxidation of the remaining seven methionines has a minimal functional effect, resulting in only modest decreases in calcium affinity and target protein binding [24]. Sequence differences near 


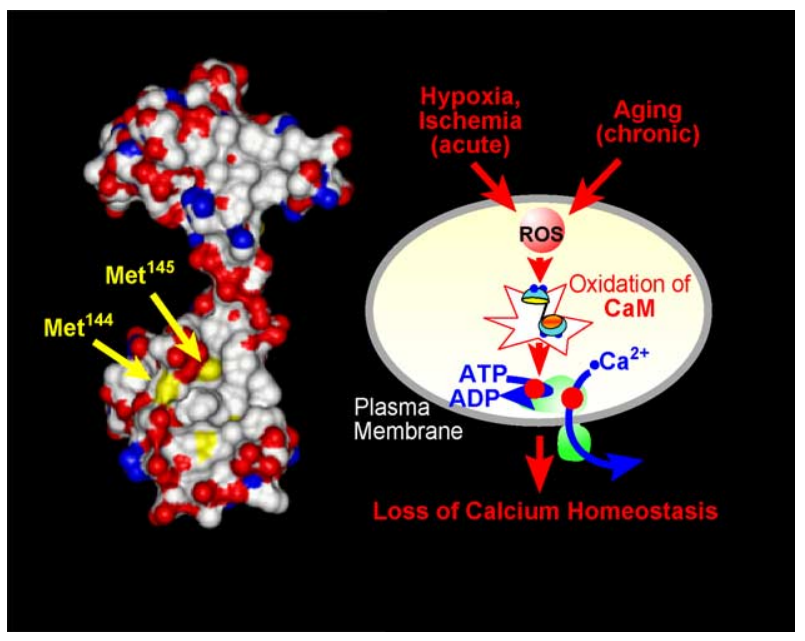

Fig. 5. CaM functions as a sensor of oxidative stress. Space filling model of CaM depicting functionally sensitive methionines (yellow) near the carboxyl-terminus (left) and proposed linkage between the generation of ROS, the oxidative modification of CaM, and inhibition of the plasma membrane (PM) Ca-ATPase that results in diminished calcium regulation and energy utilization following conditions of oxidative stress. Under acute conditions, the transient generation of oxidants such as peroxynitrite results in the selective oxidation of $\mathrm{Met}^{144}$ or $\mathrm{Met}^{145}$ [46], resulting in the rapid down-regulation of metabolism. In contrast, under chronic conditions the generation of less reactive species (e.g., hydrogen peroxide) that do not target the functionally sensitive methionines permit optimal cell function without large changes in cellular metabolism $[1,45]$. Figure adapted from that of Smallwood et al. [46].

$\mathrm{Met}^{144}$ and $\mathrm{Met}^{145}$ between species modulate the functional sensitivity to oxidative stress [45]. In this respect, all CaM sequences have very high levels of sequence identity $(91 \%$ identity between wheat and vertebrate), with most of the sequence divergence near the functionally sensitive sites proximal to the carboxyl-terminus [45], suggesting that the functional sensitivity of $\mathrm{CaM}$ to oxidation is modulated depending on the ecological niche of an organism. Thus, a comparison between the rates of oxidation of CaM isolated from vertebrate and plant sources demonstrated that the oxidation rates of the majority of methionines are essentially identical; however, there is a four-fold greater rate of oxidation of $\mathrm{Met}^{144}$ or $\mathrm{Met}^{145}$ in plant CaM that correlates with the functional sensitivity of $\mathrm{CaM}$ to oxidative stress. These results indicate large species-dependent differences in the functional sensitivities of CaM to oxidative stress. Given that the oxidation of $\mathrm{CaM}$ functions to stabilize the inhibited state of the plasma membrane Ca-ATPase (and presumably other targets as well), these results suggest an optimization of the sensitivity of CaM to oxidative stress to modulate the set point of the system (Fig. 1). Thus, in the case of vertebrates, larger amounts of nonspecific collateral damage occur prior to the down-regulation of $\mathrm{CaM}$ function in comparison to plants. Markedly different sensitivities of $\mathrm{CaM}$ to different ROS further modulate function [1]. For example, while peroxynitrite selectively oxidizes $\mathrm{Met}^{144}$ and $\mathrm{Met}^{145}$, hydrogen peroxide has a minimal effect on these functional sensors of ROS-indicating that the metabolism of different tissues will result in markedly different functional sensitivities of $\mathrm{CaM}$ to oxidative modification $[42,45,46]$. Likewise, in a comparison of different organ systems, differences in the levels of antioxidants, repair, and degradative enzymes modulate the set point of the system, whereby the accumulation of oxidized CaM functions to down-regulate metabolism and minimize the nonspecific oxidative damage to other proteins and biomolecules. Thus, under acute conditions associated with the generation of peroxynitrite, energy metabolism will be transiently down-regulated through the reversible oxidation of $\mathrm{Met}^{144}$ or $\mathrm{Met}^{145}$ in CaM, while under chronic conditions involving the generation of hydrogen peroxide minimal levels of oxidation occur at these functionally important sites $[1,45,46]$.

\subsection{Helix destabilization and global structural changes underlie oxidant-induced modulation of CaM function}

The underlying mechanism responsible for modulation of CaM function following site-specific oxidation of $\mathrm{Met}^{144}$ or $\mathrm{Met}^{145}$ permits generalizations concerning the possible roles of methionine oxidation in modulating cellular function. Oxidation of these methionines in $\mathrm{CaM}$ induces global structural changes that result in a nonproductive binding interaction that locks the plasma membrane Ca-ATPase in an inhibited state [24,30,45,47]. Thus, the oxidation of $\mathrm{Met}^{144}$ and $\mathrm{Met}^{145}$ near the middle of an amphipathic helix at the carboxyl-terminus of CaM acts to destabilize the central linker connecting the opposing domains of $\mathrm{CaM}$, functioning as a general switching mechanism to modulate calcium signaling in response to oxidative stress [23]. Thus, oxidation of $\mathrm{Met}^{144}$ or $\mathrm{Met}^{145}$ results in the structural uncoupling between the opposing domains of CaM, which favors the formation of a high-affinity inhibited enzyme complex in the case of the plasma membrane Ca-ATPase $[23,48,49]$. This nonproductive conformation of $\mathrm{CaM}_{\mathrm{ox}}$ bound to the Ca-ATPase fails to induce helix formation within the CaM-binding sequence that is required for the release of enzyme inhibition (Fig. 4).

Helix destabilization and the structural uncoupling between the opposing domains of $\mathrm{CaM}$ upon oxidation of $\mathrm{Met}^{144}$ or $\mathrm{Met}^{145}$ are consistent with other examples in the literature where the structures of methionine-rich amphipathic $\alpha$-helices are known to be sensitive to oxidation. For example, methionine sulfoxide formation results in a loss of secondary structure that interferes with helix-helix interactions to disrupt long-range stabilizing interactions between domains in proteins such as amyloid beta-peptide, HIV-2 protease, small heat shock proteins, and thrombomodulin [50-53]. Furthermore, methionine sulfoxide can disrupt the formation of higher order structures involving polymer formation in proteins such as $\alpha$-synuclein and amyloid beta-peptide [54,55]. In contrast, the methionine-rich structures around the active 
site in glutamine synthetase appear to be insensitive to oxidation [16], suggesting that in these instances methionine serves primarily as an antioxidant.

\subsection{Redox coupling of cellular metabolism through repair of oxidized CaM}

Site-specific and reversible posttranslational modifications are the hallmark of cellular signaling mechanisms, and identification of a correlation between the oxidation of a methionine in $\mathrm{CaM}$ and function suggests its physiological significance. Thus, the ability of endogenous methionine sulfoxide reductases present in all organisms to reduce (i.e., repair) oxidized methionines indicates that following an acute oxidative stress, oxidation of $\mathrm{CaM}$ is likely to be transient [42,56-61]. Methionine sulfoxide reductases exist in two forms (i.e., MsrA and MsrB) that mediate the steriospecific reduction of $\mathrm{S}$ and $\mathrm{R}$ sterioisomers of methionine sulfoxide, respectively [60]. Together, MsrA and $\mathrm{MsrB}$, in conjunction with electron donors NADPH and thioredoxin, are able to fully reduce oxidized $\mathrm{CaM}$ and restore function $[58,60]$. Regulation of rates of repair is therefore tightly linked to cellular redox conditions. Thus, when ROS exceed antioxidant cellular defense mechanisms, cellular NADPH and reduced thioredoxin levels decrease and result in the inactivation of methionine sulfoxide reductases. Under these conditions, $\mathrm{CaM}_{\mathrm{ox}}$ accumulation will result in down-regulation of metabolism and a corresponding decrease in the generation of ROS through respiratory control mechanisms. Following restoration of cellular redox conditions, repair of oxidized methionines occurs with a restoration of protein function. Alternatively, under chronic conditions of oxidative stress, selective degradation of $\mathrm{CaM}_{\mathrm{ox}}$ by the proteasome will function to lower cellular $\mathrm{CaM}$ concentrations with an associated decrease in metabolic rates [41,62]. In conclusion, CaM oxidation represents an important switch point at the first step of the calcium signal to modulate metabolism and orchestrate an adaptive cellular response through transient changes in activity or through the down-regulation of $\mathrm{CaM}$ levels.

\section{Nitration as an indicator of peroxynitrite: a potent oxidant of methionines}

Peroxynitrite $\left(\mathrm{ONOO}^{-}\right)$, formed from the spontaneous combination of nitric oxide (NO) and superoxide $\left(\mathrm{O}_{2}^{-}\right)$, is among the most common physiological oxidants $[63,64]$. The near diffusion-controlled rate of this reaction competes effectively with removal of superoxide by SOD, with the effect that $\mathrm{ONOO}^{-}$formation occurs when cellular nitric oxide levels are comparable to those of SOD (i.e., micromolar). Diffusive and capable of rapidly crossing lipid membranes, peroxynitrite is highly reactive towards proteins, lipids and nucleic acids [65].
Within proteins, methionine and cysteine oxidation are common results of peroxynitrite exposure, as well as the nitration of tyrosines. Sulfur oxidations are suggested to involve a two-electron oxidation by peroxynitrous acid $(\mathrm{ONOOH})$; with a $\mathrm{p} K_{\mathrm{a}}$ of 6.8 , the protonated form of peroxynitrite is abundantly available at intracellular $\mathrm{pH}$ [66]. On the other hand, tyrosine nitration involves the $\mathrm{CO}_{2}$ adduct of peroxynitrite, i.e., nitrosoperoxo-carbonate $\left(\mathrm{ONOOCO}_{2}^{-}\right)$which is abundant at physiological levels of carbon dioxide/bicarbonate [66-68]). In metabolically active tissues, such as respiring muscle, induced changes in $\mathrm{pH}$ and carbon dioxide levels may determine the balance between tyrosine nitration and oxidation of methionines or cysteines. The development of an antibody reactive against nitrotyrosine modifications within proteins has allowed facile detection of this signature of reactive nitrogen species (RNS); as a result, nitrotyrosine-modified proteins have received more attention than other products of peroxynitrite such as methionine sulfoxides [69]. Nevertheless, methionine oxidation is present in samples that are immunoreactive against nitrotyrosine antibodies, making it problematic to differentiate between the functional effects of these oxidative and nitrative modifications.

\subsection{Nitration of the SR Ca-ATPase (SERCA2a)}

In skeletal muscle, nitration of the SERCA Ca-ATPase, a highly abundant SR protein, indicates the presence of peroxynitrite within the myocyte. Alternative nitrating species have been proposed, both in vitro and in vivo, e.g., mechanisms involving peroxidases in inflammatory cells [70]. However, the involvement of peroxynitrite in the nitration of SERCA is consistent with the expression of all three nitric oxide synthase isoforms (NOS I, II, III) within the myocyte and the measured generation of both superoxide and nitric oxide in muscle as a result of contractile activity [71-73].

The essential role of the Ca-ATPase in controlling properties of the calcium transient suggests its possible role as an additional sensor of oxidative stress working in concert with $\mathrm{CaM}$ in muscle. Therefore, the nitration of the Ca-ATPase and the oxidation of methionine within its regulator protein, phospholamban, will be discussed in view of its implications for stress response in aging muscle.

The SERCA Ca-ATPase is the major determinant of the calcium transient in muscle. The sarcoplasmic reticulum (SR) $\mathrm{Ca}^{2+}-\mathrm{Mg}^{2+}$-ATPase (Ca-ATPase) actively transports calcium ions away from relaxing contractile fibers in the cytosol into the lumen of the SR after each contractile event, thereby resetting the 1000-fold calcium gradient across the SR membrane. In addition to its immediate regulation of muscle contraction, changes in amplitude and duration of the calcium transient can be translated into longer-term changes in myocyte properties through gene transcription. For example, calcium transients, through CaM-kinase II and calcineurin regulation of the phosphorylation state of the 
transcription factor NFAT drive processes that include fiber type switching in skeletal muscle, exercise-induced changes, changes leading to cardiac hypertrophy, and developmental changes in both heart and skeletal muscle [8,74-78].

Nitration of SERCA2a results in functional inactivation in aging muscle. Chronologically, one of the first deficits observed during aging of skeletal muscle is a prolongation of the calcium transient with concomitantly longer times of muscle relaxation $[79,80]$. The basis of these changes is the age-related decreases in rates of calcium transport coupled with ATP hydrolysis by the SR Ca-ATPase [21,22,81-83]. This loss of Ca-ATPase activity, in turn, correlates with accumulation of 3-nitrotyrosine-modified forms of SERCA2a, the isoform expressed in slow twitch muscle; modification of other amino acids does not occur at stoichiometric levels, with the exception of an increased oxidation of 1.5 mol cysteine per SERCA2a. However, this oxidation occurs early in the life span without an associated effect on Ca-ATPase activity [22].

The increase in nitrotyrosine modification of SERCA2a with aging is substantial, where the SERCA2a isoform of the Ca-ATPase is associated with $4 \mathrm{~mol}$ nitrotyrosine per mol of Ca-ATPase, increased from approximately equimolar ratios of nitrotyrosine per Ca-ATPase in the young adult muscle of the Fischer 344 rat. In vitro exposure of SR membranes from young adult muscle to varying amounts of peroxynitrite, which induces progressive formation of nitrotyrosine up to $4 \mathrm{~mol}$ per mole of Ca-ATPase, correlates with a progressive inhibition of Ca-ATPase activity. Thus, these experiments provide strong evidence to indicate that the in vivo increases in nitration observed during aging form the basis for the observed loss of Ca-ATPase activity.

Nitration of SERCA2a appears to be a general marker of nitrative stress in muscle. For example, nitration of SERCA2a has also been observed in aging rat heart, in ischemic human heart, and in hypercholestemic aortic smooth muscle [84-86]. The presence of peroxynitrite in the myocyte, a consequence of elevated levels of nitric oxide in contracting muscle, suggests that decreased activities of other muscle proteins with functionally important cysteines and methionines may also contribute to loss of function in aged cells. In view of the prolonged calcium release step in aging muscle, modification of the cysteine-rich ryanodine receptor calcium release channel or of methionines within its major regulator, $\mathrm{CaM}$, are likely to be relevant. A recent proteomic screen demonstrated the oxidation of $\mathrm{Met}^{20}$ of phospholamban in (nonfailing) human heart [87].

\subsection{Sites of nitrotyrosine suggest a functional mechanism}

Among the 18 tyrosines within the SERCA2a sequence, at least three are nitrated in senescent skeletal muscle; these have been identified [22]. These consist of $\mathrm{Tyr}^{753}$, which is present in young adult muscle and the vicinal tyrosines, $\mathrm{Tyr}^{294}$ and $\mathrm{Tyr}^{295}$, which appear in their nitrated form only in senescent skeletal muscle. Based on the available crystal structures of the highly homologous SERCA1 isoform of this protein, the location of these sites within SERCA2a can be inferred and indicates their buried positions within the Ca-ATPase structure (Fig. 3A), suggesting the possibility that modified sites are not accessible to repair or degradative enzymes. The vicinal tyrosines, $\mathrm{Tyr}^{294}$ and $\mathrm{Tyr}^{295}$, lie on a membrane spanning helix near the interface of the Ca-ATPase with the SR lumen (Fig. 3); this position is consistent with the diffusive nature of peroxynitrite, which is capable of rapid diffusion across lipid bilayers [65]. These vicinal tyrosines are the most functionally relevant, which is apparent from their location in a functionally critical region of the Ca-ATPase, on a helix that provides several ligands for calcium binding and is co-linear with the phosphorylation site of the enzyme. Moreover, this region represents a pivot point, which must accommodate large angular changes in transmembrane helices during the transport cycle [88]. Thus, nitration of two closely apposed tyrosines is likely to distort the local protein structure due to the additional bulk of $\mathrm{NO}_{2}$ groups on the phenolic ring. In addition, the decreased $\mathrm{p} K_{\mathrm{a}}$ of nitrotyrosine ( 7.2) suggests the deprotonation of these nitrotyrosines with the accompanying electrostatic repulsion of two negative charges that may constrain structural rearrangements important to transport.

\subsection{Isoform selectivity of nitration and inhibitory role of phospholamban}

A striking characteristic of the nitration of the $\mathrm{Ca}$ ATPase (SERCA) in muscle is its specificity in vivo for the SERCA2a isoform of the Ca-ATPase, expressed in slow twitch skeletal muscle, in preference for the highly homologous SERCA1 form, expressed in fast twitch skeletal muscle. In vitro exposure to peroxynitrite demonstrates that SERCA1 is inherently less sensitive to nitration than is SERCA2a. Indeed, SERCA1 can be characterized as cysteine reactive under the same conditions of peroxynitrite exposure that result in nitration of SERCA2a [89]. In addition to the high extent of sequence similarity (84\% identical), 16 of the 18 Tyr present within SERCA2a are conserved sites with respect to the SERCA1 sequence; the latter isoform has a total of 22 Tyr [90]. Moreover, the amino acid sequences surrounding nitrotyrosine sites identified in skeletal muscle SERCA2a, i.e., $\mathrm{Tyr}^{294}$, $\mathrm{Tyr}^{295}$, and $\mathrm{Tyr}^{753}$, are identical with those in SERCA1, which are not sensitive to nitration. Cysteine residues are also highly conserved between these two isoforms. This perplexing difference in oxidation characteristics between highly similar isoforms is difficult to assess in the absence of an atomic resolution structure for SERCA2a. However, a striking difference between these isoforms is the in vivo coexpression of SERCA2a with the inhibitory protein 
phospholamban [91]. This 52-amino-acid membrane-spanning phosphoprotein interacts with the Ca-ATPase to inhibit function, resulting in a reduced calcium sensitivity (Fig. 3A). Inhibition is released following phosphorylation of either $\operatorname{Ser}^{16}$ by PKA or $\mathrm{Thr}^{17}$ by CaM-dependent protein kinase II, permitting in vivo responses to $\beta$ adrenergic stimulation. Furthermore, phospholamban binding to SERCA stabilizes helical structures and induces a reorientation of the nucleotide binding domain, thereby restricting catalytically important motions [92-94]. Therefore, interaction with phospholamban may induce a conformation of SERCA2a in which either tyrosines are more accessible for nitration or reactive cysteines are blocked, allowing the slower reacting tyrosines to be modified [95]. Regardless of the specific mechanism, the presence of a nitration-sensitive form of SERCA in a mitochondria-rich oxidative cellular environment, such as slow twitch skeletal muscle and heart, ensures the ability of the myocyte for down regulation of energy metabolism during an oxidative/nitrative stress.

\section{Phospholamban and Ca-ATPase inhibition: linkage between methionine oxidation and calcium uptake}

A recent proteomic screen of phosphoproteins has revealed the oxidation of $\mathrm{Met}^{20}$ within phospholamban in nonfailing human heart following trauma, consistent with the presence of an oxidative/nitrative stress in the heart by peroxynitrite (Fig. 6) [87]. This observation also raises the intriguing question regarding what functional significance this oxidation may have on phospholamban regulation of SERCA2a and its possible role as an alternate sensor of cellular stress. Certain predictions can be made based on our knowledge of the mechanism underlying the regulation of the normal function of the Ca-ATPase.

\subsection{Phospholamban regulates the Ca-ATPase}

Phospholamban is a 52-amino-acid phosphoprotein having a single transmembrane helix and an N-terminal cytosolic helix connected by a flexible loop region $\left(\mathrm{TIEM}_{20}\right)$, which acts as a conformational switch when phospholamban is phosphorylated at $\operatorname{Ser}^{16}$ by cAMPdependent protein kinase (PKA) or at $\mathrm{Thr}^{17}$ by CaM-kinase II, relieving inhibitory interactions between phospholamban and SERCA2a of SERCA2a (Fig. 3A). These two phosphorylation sites appear to serve as alternate switches originating from different signal pathways; one phosphorylation per phospholamban provides full activation of SERCA with no additional effect from dual phosphorylation [96]. Activation of calcium transport is manifested by a shift in the calcium concentration dependence of enzyme activation to lower calcium concentrations; it is not calcium affinity that is modulated by phospholamban, but rather the conformational change of the Ca-ATPase subsequent to calcium binding [97]. The nature of that conformational change requires an understanding of the mechanism of active calcium transport by the Ca-ATPase in the absence of phospholamban. Virtually all of the detailed information, available to date, originates from structural and kinetic studies of the SERCA1 isoform, which by all indications undergoes similar or identical kinetics and mechanism of action relative to SERCA2a [98].

\subsection{Mechanism of active calcium transport by the Ca-ATPase}

Integral to the mechanism of calcium transport is its coupling through the protein structure to the distal sites of ATP hydrolysis, which provides the chemical energy for transport. This process is mediated through transfer of the $\gamma$-phosphoryl group of ATP bound to the N-domain to

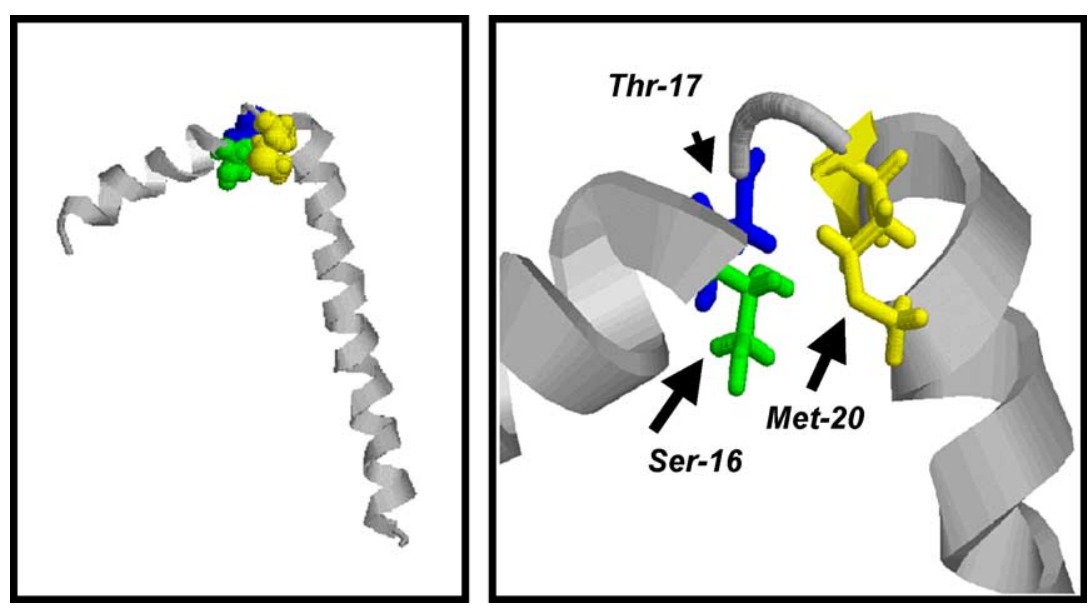

Fig. 6. Structure of phospholamban. Methionine sulfoxide at $\mathrm{Met}^{20}$, shown in yellow, lies proximal to sites phosphorylated by cAMP-dependent protein kinase (PKA) (Ser ${ }^{16}$; green) or calmodulin-dependent protein kinase II (Thr ${ }^{17}$; blue) in switch region. The left panel shows a model of the entire phospholamban protein; the right panel shows a close view of the flexible loop TIEM ${ }^{20}$. Illustration was prepared using the coordinates 1 FJK.pdb and the program RASMOL [114,115]. 
covalent binding at $\mathrm{Asp}^{351}$ in the P-domain (Fig. 3A); the resulting phosphorylated intermediate is the hallmark characteristic of SERCA and other E-P type ion transport proteins. From high-resolution crystal structures in combination with dynamic measurements of SERCA in solution, it is clear that calcium transport requires collaboration of the entire protein structure. However, while conformational changes within the transmembrane domain are restricted by the bilayer, large amplitude motions of the cytoplasmic domains facilitate their substantial angular and spatial rearrangements that juxtapose ATP bound to the $\mathrm{N}$ (nucleotide binding)-domain with $\mathrm{Asp}^{351}$ within the P(phosphorylation)-domain (Fig. 3A) [88,99-102]. Thus, Brownian diffusion of protein domains plays an important role in the enzyme phosphorylation necessary for calcium transport.

\subsection{Mechanism of inhibition (and activation) by phospholamban}

Phospholamban regulates the Ca-ATPase with formation of a complex with the Ca-ATPase with several sites of interaction identified by cross-linking reagents and site-directed mutagenesis, which are located within both transmembrane and cytosolic domains (Fig. 3A). Specific sequences within the cytoplasmic N-domain $\left(\mathrm{DKKDPVK}^{402}\right.$ ) and within the cytosolic helix of phospholamban (Glu2 to Ile18) have been identified as critical to the inhibitory interactions of nonphosphorylated phospholamban [103-105]. Phosphorylated phospholamban, mediating activation of the Ca-ATPase, retains its association with the Ca-ATPase, but with a redistribution of protein-protein contacts [106-109]. Phosphorylation of bound phospholamban results in a slight shortening of the cytosolic region of phospholamban, consistent with $\alpha$-helical stabilization by the salt bridge between the phosphoryl group at $\mathrm{Ser}^{16}$ and the guanidino group of $\operatorname{Arg}^{13}$ [109,110].

When phospholamban binds to the Ca-ATPase, its inherent flexibility, as measured by its rotational dynamics and distance measurements from the N-terminal face of the transmembrane helix (position 24) to the N-terminal side of the cytoplasmic helix (position 6), is severely restricted, indicating tight binding and modification of the orientation and motional range of $\mathrm{N}$-domain diffusion [106,110,111]. Thus, the kinetics of productive transfer of the $\gamma$-phosphoryl group of ATP bound to the N-domain to $\mathrm{Asp}^{351}$ in the Pdomain are diminished by the tight interaction with phospholamban at sites on the N-domain of the ATPase (Fig. 3). Phosphorylation of phospholamban releases the inhibitory interactions with the N-domain of the Ca-ATPase (Fig. 7). This mechanism of phospholamban regulation of SERCA is reminiscent of the regulation of the PMCA through the inhibitory domain, except that CaM binding, rather than phosphorylation, is the trigger for activation of the plasma membrane Ca-ATPase.

\subsection{Predicted effect of oxidation of $\mathrm{Met}^{20}$ within phospholamban on Ca-ATPase activation}

Addition of an oxygen atom to the sulfur atom of methionine to form methionine sulfoxide results in both increased polarity and disruption of helical structures. The latter effect has been demonstrated to provide the functionally relevant changes upon oxidation of methionines within $\mathrm{CaM}$. Thus, oxidation of $\mathrm{Met}^{20}$ at the interface with the critical switch region of phospholamban is expected to induce disorder within the N-terminal region of the transmembrane helix of phospholamban, reducing the ability of

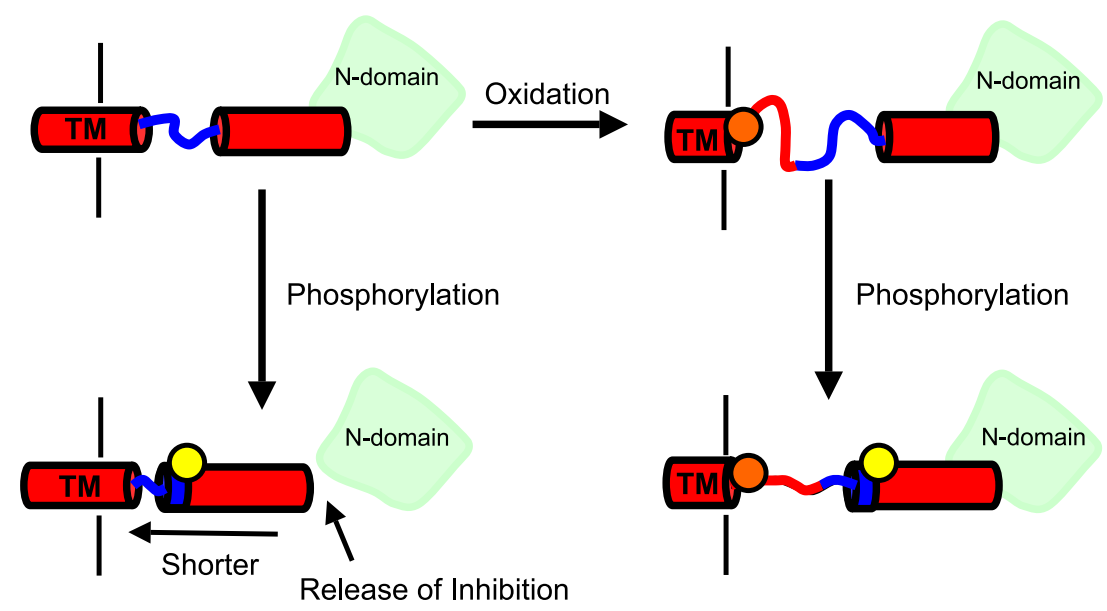

Fig. 7. Release of SERCA Ca-ATPase inhibition requires shortening of interdomain switch region in phospholamban. Phosphorylation (yellow circle) of Ser ${ }^{16}$ in phospholamban induces helix formation (blue cylinder) with a concomitant shortening of the cytoplasmic portion of phospholamban and release of the inhibitory interaction between phospholamban (red) and the N-domain (green) of the Ca-ATPase $[109,110,111]$. This conformational switch is relaxed following helical destabilization upon $\mathrm{Met}^{20}$ oxidation (orange), alleviating physical constraint imposed to maintain inhibitory interaction between phospholamban and the $\mathrm{N}$-domain of the Ca-ATPase following phosphorylation, consistent with the reported insensitivity to $\beta$-adrenergic signaling upon generation of peroxynitrite [116]. The transmembrane helix (TM) functions as a fixed point that defines the overall dimensions of the cytosolic portion of phospholamban. 
phosphorylation-induced shortening of the cytosolic region of phospholamban to release inhibitory interactions with the $\mathrm{N}$-domain of the ATPase (Figs. 5 and 7). As in the case of the inhibitory domain of the plasma membrane Ca-ATPase, which does not dissociate from the N-domain of the PMCA in the presence of oxidized CaM; upon oxidation of $\mathrm{Met}^{20}$ the phosphorylated phospholamban can no longer dissociate from the N-domain of SERCA, effectively blocking $\beta$ adrenergic signaling and locking the Ca-ATPase in the inhibited state (Fig. 3).

\section{Methionine oxidation functions as a conformational switch}

The sensitivity of methionines to oxidation, coupled with large differences in the helix-promoting abilities of methionine and the resulting methionine sulfoxide, permits their use as sensors of oxidative stress to serve as conformational switches that modulate the activity of central regulatory proteins (i.e., Ca-ATPase and the regulatory proteins phospholamban and $\mathrm{CaM}$ ) (Fig. 8). These conformational switches are directly coupled to cellular redox conditions through the actions of methionine sulfoxide reductases, which function to couple reducing equivalents from thioredoxin and NADPH to reduce surface exposed methionine sulfoxides. Thus, the reversible oxidation of methionines in critical regulatory proteins functions as a rheostat of cellular metabolism to maintain optimal cell function with minimal levels of nonspecific oxidative damage to other biomolecules that can result in irreversible cell damage.

Whereas addition of an oxygen to the methionine sidechain results in its increased polarity, this change has only a minor effect on the global protein structure. The primary physical basis underlying the oxidation-induced structural switching associated with methionine sulfoxide is that this modified amino acid is particularly unsuited to helices, while methionine is well favored. The ability of methionines to stabilize helices is related to their extended and flexible conformation, which is quite distinct from those of the more bulky aliphatic side chains associated with Val and Ile whose branch points at the $\beta$-carbon place additional constraints on their conformations [112]. Thus, Met side chains extend far from the helical backbone, and permit favorable interactions with neighboring helices to stabilize tertiary structural interactions. Furthermore, the sulfur in Met presents a hydrogen-bonding capability that further differentiates this amino acid from its more bulky cousin Leu in facilitating the stabilization of helices. Thus, methionines are often found near the center of helices at interfacial contact points that stabilize protein structures. Upon methionine oxidation, helices are destabilized, in part, because of a large reduction in the conformational flexibility of the side chain. This can be understood in analogy to the destabilizing influence associated with the introduction of Ser or Thr into $\alpha$-helices.

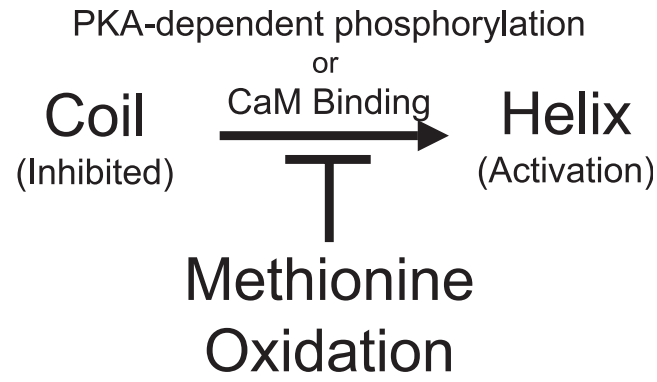

Fig. 8. Methionine oxidation blocks release of inhibitory interaction through helix destabilization. Activation of Ca-ATPase function through the release of the inhibitory interaction between either phospholamban or the inhibitory domain of the plasma membrane Ca-ATPase and the Ndomain requires helix formation and associated physical shortening of cytosolic portions of proteins, which is blocked by methionine oxidation. Levels of methionine oxidation are modulated by cellular antioxidant defenses and repair enzymes, providing a functional linkage between cellular reducing conditions and energy utilization through Ca-ATPase activation.

Indeed, Ser is one of the most unfavorable residues in a helix due to the ability of hydrogen-bonding interactions involving the oxygen to induce stabilizing interactions that favor turns, sheets, and disordered structures [112].

\section{Acknowledgements}

This work was supported by grants AG12993, AG18013 and AG17996 from the National Institutes of Health.

\section{References}

[1] T.C. Squier, D.J. Bigelow, Protein oxidation and age-dependent alterations in calcium homeostasis, Front. Biosci. 5 (2000) D504-D526.

[2] C.-K. Lee, D.B. Allison, J. Brand, R. Weindruch, T.A. Prolla, Transcriptional profiles associated with aging and middle age-onset caloric restriction in mouse hearts, Proc. Natl. Acad. Sci. U. S. A. 99 (2002) 14988-14993.

[3] S.D. Hursting, J.A. Lavigne, D. Berrigan, S.N. Perkins, J.C. Barrett, Calorie restriction, aging, and cancer prevention: mechanisms of action and applicability to humans, Annu. Rev. Med. 54 (2003) $131-152$.

[4] A.J. Dirks, C. Leeuwenburgh, Aging and lifelong calorie restriction result in adaptations of skeletal muscle apoptosis repressor, apoptosis-inducing factor, $\mathrm{X}$-linked inhibitor of apoptosis, caspace3, and caspace-12, Free Radic. Biol. Med. 36 (2004) 27-39.

[5] S. Blanc, D. Schoeller, J. Kemnitz, R. Weindruch, R. Colman, W. Newton, K. Wink, S. Baum, J. Ramsey, Energy expenditure of rhesus monkeys subjected to 11 years of dietary restriction, J. Clin. Endocrinol. Metab. 88 (2003) 16-23.

[6] K. Deisseroth, E.K. Heist, R.W. Tsien, Translocation of calmodulin to the nucleus supports CREB phosphorylation in hippocampal neurons, Nature 392 (1998) 198-202.

[7] A. Antonsson, K. Hughes, S. Edin, T. Grundstrom, Regulation of cRel nuclear localization by binding of $\mathrm{Ca}^{2+} /$ calmodulin, Mol. Cell. Biol. 23 (2003) 1418-1427.

[8] R.A. Schulz, K.E. Yutzey, Calcineurin signaling and NFAT activation in cardiovascular and skeletal muscle development, Dev. Biol. 266 (2004) $1-16$. 
[9] T.J. Weber, S. Negash, H.S. Smallwood, K.S. Ramos, B.D. Thrall, T.C. Squier, Calmodulin involvement in stress-activated nuclear localization of albumin in JB6 epithelial cells, Biochemistry 43 (2004) 7443-7450.

[10] K.L. Yap, J. Kim, J.K. Truong, M. Sherman, T. Yuan, M. Ikura, Calmodulin target database, J. Struct. Funct. Genomics 1 (2000) $8-14$.

[11] A.M. Katz, Physiology of the Heart, 3rd ed., Lippincott, Williams and Wilkins, New York, 2001.

[12] T.C. Squier, Oxidative stress and protein aggregation during biological aging, Exp. Gerontol. 36 (2001) 1539-1550.

[13] J.P. Blass, R.K. Sheu, G.E. Gibson, Inherent abnormalities in energy metabolism in Alzheimer disease. Interaction with cerebrovascular compromise, Ann. N.Y. Acad. Sci. 903 (2000) 204-221.

[14] L.J. Yan, R.S. Sohal, Mitochondrial adenine nucleotide translocase is modified oxidatively during aging, Proc. Natl. Acad. Sci. U. S. A. 95 (1998) 12896-12901.

[15] A. Gafni, Structural modifications of proteins during aging, J. Am. Geriatr. Soc. 45 (1997) 871-880.

[16] R.L. Levine, L. Mosoni, B.S. Berlett, E.R. Stadtman, Methionine residues as endogenous antioxidants in proteins, Proc. Natl. Acad. Sci. U. S. A. 93 (1996) 15036-15040.

[17] R. Moreau, S.H. Heath, C.E. Doneanu, J.G. Lindsay, T.M. Hagen, Age-related increase in 4-hyroxynonenal adduction to rat heart alpha-ketoglutarate dehydrogenase does not cause loss of its catalytic activity, Antioxid. Redox Signal. 5 (2003) 517-527.

[18] J.P. Eu, J. Sun, L. Xu, J.S. Stamler, G. Meissner, The skeletal muscle calcium release channel: coupled $\mathrm{O}_{2}$ sensor and NO signaling functions, Cell 102 (2000) 499-509.

[19] Y. Yao, D. Yin, G.S. Jas, K. Kuczera, T.D. Williams, Ch. Schoneich, T.C. Squier, Oxidative modification of a carboxyl-terminal vicinal methionine in calmodulin by hydrogen peroxide inhibits calmodulindependent activation of the plasma membrane Ca-ATPase, Biochemistry 35 (1996) 2767-2787.

[20] J. Gao, D. Yin, Y. Yao, H. Sun, Z. Qin, Ch. Schoneich, T.D. Williams, T.C. Squier, Loss of conformational stability in calmodulin upon methionine oxidation, Biophys. J. 74 (1998) 1115-1134.

[21] R.I. Viner, D.A. Ferrington, G.I. Aced, M. Miller-Schlyer, D.J. Bigelow, Ch. Schoneich, In vivo aging of rat skeletal muscle sarcoplasmic reticulum Ca-ATPase. Chemical analysis and quantitative simulation by exposure to low levels of peroxyl radicals, Biochim. Biophys. Acta 1239 (1997) 321-335.

[22] R.I. Viner, D.A. Ferrington, T.D. Williams, D.J. Bigelow, Ch. Schoneich, Protein modification during biological aging: selective tyrosine nitration of the SERCA2a isoform of the sarcoplasmic reticulum Ca-ATPase, Biochem. J. 340 (1999) 657-659.

[23] J. Gao, Y. Yao, T.C. Squier, Oxidatively modified calmodulin binds to the plasma membrane Ca-ATPase in a non-productive and conformationally disordered complex, Biophys. J. 80 (2001) $1791-1801$

[24] R.K. Bartlett, R.J. Bieber Urbauer, A. Anbanandam, H.S. Smallwood, J.L. Urbauer, T.C. Squier, Oxidation of $\mathrm{Met}^{144}$ and $\mathrm{Met}^{145}$ in calmodulin blocks calmodulin dependent activation of the plasma membrane Ca-ATPase, Biochemistry 42 (2003) 3231-3238.

[25] E.M. Balog, L.E. Norton, R.A. Bloomquist, R.L. Cornea, D.J. Black, C.F. Louis, D.D. Thomas, B.R. Fruen, Calmodulin oxidation and methionine to glutamine substitutions reveal methionine residues critical for functional interaction with ryanodine receptor-1, J. Biol. Chem. 278 (2003) 15615-15621.

[26] H.J. Montgomery, R. Bartlett, B. Perdicakis, E. Jervis, T.C. Squier, J.G. Guillemette, Activation of constitutive nitric oxide synthases by oxidized calmodulin, Biochemistry 42 (2003) 7759-7768.

[27] A. Persechini, P.M. Stemmer, Calmodulin is a limiting factor in the cell, Trends Cardiovasc. Med. 12 (2002) 32-37.

[28] Q.K. Tran, D.J. Black, A. Persechini, Intracellular coupling via limiting calmodulin, J. Biol. Chem. 278 (2003) 24247-24250.
[29] A. Persechini, B. Cronk, The relationship between the free concentrations of $\mathrm{Ca}^{2+}$-calmodulin in intact cells, J. Biol. Chem. 274 (1999) 6827-6830.

[30] Y. Yao, J. Gao, T.C. Squier, Dynamic structure of the calmodulin-binding domain of the plasma membrane Ca-ATPase in native erythrocyte ghost membranes, Biochemistry 35 (1996) 12015-12028.

[31] J. Martin-Nieto, A. Villalobo, The human epidermal growth factor receptor contains a juxtamembrane calmodulin-binding site, Biochemistry 37 (1998) 227-236.

[32] J.A. Clapperton, S.R. Martin, S.J. Smerdon, S.J. Gamblin, P.M Bayley, Structure of the complex of calmodulin with the target sequence of calmodulin-dependent protein kinase I: studies of the kinase activation mechanism, Biochemistry 41 (2002) $14669-14679$.

[33] D.B. Sacks, B. Mazus, J.L. Joyal, The activity of calmodulin is altered by phosphorylation: modulation of calmodulin function by the site of phosphate incorporation, Biochem. J. 312 (1995) 197-204.

[34] D.B. Sacks, M.M. Lopez, Z. Li, D. Kosk-Kosicka, Analysis of phosphorylation and mutation of tyrosine residues of calmodulin on its activation of the erythrocyte $\mathrm{Ca}^{2+}$-transporting ATPase, Eur. J. Biochem. 239 (1996) 98-104.

[35] D.M. Grief, D.B. Sacks, T. Michel, Calmodulin phosphorylation and modulation of endothelial nitric oxide synthase catalysis, Proc. Natl. Acad. Sci. U. S. A. 101 (2004) 1165-1170.

[36] X. Wang, V.C. Culotta, C.B. Klee, Superoxide dismutase protect calcineurin from inactivation, Nature 383 (1996) 434-437.

[37] D. Namgaladze, H.W. Hofer, V. Ullrich, Redox control of calcineurin by targeting the binuclear $\mathrm{Fe}^{2+}-\mathrm{Zn}^{2-}$ center at the enzyme active site, J. Biol. Chem. 277 (2002) 5962-5969.

[38] K.D. Osborn, A. Zaidi, A. Mandal, R.J. Bieber Urbauer, C.K. Johnson, Single-molecule dynamics of the calcium-dependent activation of plasma-membrane $\mathrm{Ca}^{2+}$-ATPase by calmodulin, Biophys. J. 87 (2004) 1892-1899.

[39] S. Vougier, J. Mary, N. Dautin, J. Vinh, B. Friguet, D. Ladant, Essential role of methionine residues in calmodulin binding to Bordetella pertussis adenylate cyclase, as probed by selective oxidation and repair by the peptide methionine sulfoxide reductases, J. Biol. Chem. 279 (2004) 30210-30218.

[40] E. Tarcsa, G. Szymanska, S. Lecker, C.M. O'Connor, A.L. Goldberg, $\mathrm{Ca}^{2+}$-free calmodulin and calmodulin damaged by in vitro aging are selectively degraded by $26 \mathrm{~S}$ proteasomes without ubiquitination, J. Biol. Chem. 275 (2000) 20295-20301.

[41] D.A. Ferrington, H. Sun, K.K. Murray, J. Costa, T.D. Williams, D.J. Bigelow, T.C. Squier, Selective degradation of oxidized calmodulin by the 20S proteasome, J. Biol. Chem. 276 (2001) 937-943.

[42] J. Gao, D. Yin, Y. Yao, T.D. Williams, T.C. Squier, Progressive decline in the ability of calmodulin isolated from aged brain to activate the plasma membrane Ca-ATPase, Biochemistry 37 (1998) 9536-9548.

[43] D.A. Ferrington, X. Chen, A.G. Krainev, E.K. Michaelis, D.J. Bigelow, Protein half-lives of calmodulin and the plasma membrane Ca-ATPase in rat brain, Biochem. Biophys. Res. Commun. 237 (1997) $163-165$

[44] R. Bronson, D. Birt, S.N. Meydani, Biomarkers as early predictors of long-term health status and human immune function, Nutr. Rev. 57 (1999) S7-S12.

[45] D. Yin, K. Kuczera, T.C. Squier, The sensitivity of carboxyl-terminal methionines in calmodulin isoforms to oxidation by $\mathrm{H} 2 \mathrm{O} 2$ modulates the ability to activate the plasma membrane Ca-ATPase, Chem. Res. Toxicol. 13 (2000) 103-110.

[46] H.S. Smallwood, N.A. Galeva, R.K. Bartlett, R.J. Bieber Urbauer, T.D. Williams, J.L. Urbauer, T.C. Squier, Selective nitration of Tyr ${ }^{99}$ in calmodulin as a marker of cellular conditions of oxidative stress, Chem. Res. Toxicol. 16 (2003) 95-102. 
[47] D. Yin, H. Sun, R.F. Weaver, T.C. Squier, Nonessential role for methionines in the productive association between calmodulin and the plasma membrane Ca-ATPase, Biochemistry 38 (1999) $13654-13660$.

[48] H. Sun, D. Yin, T.C. Squier, Calcium-dependent structural coupling between opposing globular domains of calmodulin involves the central helix, Biochemistry 38 (1999) 12266-12279.

[49] H. Sun, D. Yin, L.A. Coffeen, M.A. Shea, T.C. Squier, Mutation of Tyr138 disrupts the structural coupling between the opposing domains in vertebrate calmodulin, Biochemistry 40 (2001) 9605-9617.

[50] A.A. Watson, D.P. Fairlie, D.J. Craik, Solution structure of methionine-oxidized amyloid $\beta$-peptide (1-4). Does oxidation affect conformational switching? Biochemistry 37 (1998) 12700-12706.

[51] D.A. Davis, F.M. Newcomb, J. Moskovitz, P.T. Wingfield, S.J. Stahl, J. Kaufman, H.M. Fales, R.L. Levine, R. Yarchoan, HIV-2 protease is inactivated after oxidation at the dimer interface and activity can be partly restored with methionine sulfoxide reductase, Biochem. J. 346 (2000) 305-311.

[52] U. Härndahl, B.P.A. Kokke, N. Gustavsson, S. Linse, K. Berggren, F. Tjerneld, W.C. Boelens, C. Sundby, Thechaperone-like activity of a small heat shock protein is lost after sulfoxidation of conserved methionines in a surface-exposed amphipathic $\beta$-helix, Biochim. Biophys. Acta 1545 (2001) 227-237.

[53] M.J. Wood, A. Becvar, J.H. Prieto, G. Melacini, E.A. Komives, NMR structures reveal how oxidation inactivates thrombomodulin, Biochemistry 42 (2003) 11932-11942.

[54] C. Garcia-Echeverria, Disruption of coiled coil formation by methionine oxidation, Bioorg. Med. Chem. Lett. 6 (1996) 229-232.

[55] M.J. Hokenson, V.N. Uversky, J. Goers, G. Yamin, L.A. Munishkina, A.L. Fink, Role of individual methionines in the fibrillation of methionine-oxidized $\beta$-synuclein, Biochemistry 43 (2004) 4621-4633.

[56] J. Moskovitz, S. Bar-Noy, W.M. Williams, J. Requena, B.S. Berlett, E.R. Stadtman, Methionine sulfoxide reductase (MsrA) is a regulator of antioxidant defense and life span in mammals, Proc. Natl. Acad. Sci. U. S. A. 98 (2001) 12920-12925.

[57] V.S. Sharov, D.A. Ferrington, T.C. Squier, Ch. Schöneich, Diastereoselective reduction of protein-bound methionine sulfoxide by methionine sulfoxide reductase, FEBS Lett. 455 (1999) $247-250$.

[58] H. Sun, J. Gao, D.A. Ferrington, H. Biesiada, T.D. Williams, T.C. Squier, Repair of oxidized calmodulin by methionine sulfoxide reductase restores the ability to activate the plasma membrane CaATPase, Biochemistry 38 (1999) 105-112.

[59] T. Hoshi, S.H. Heinemann, Regulation of cell function by methionine oxidation and reduction, J. Physiol. 531 (2001) 1-11.

[60] R. Grimaud, B. Ezraty, J.K. Mitchell, D. Lafitte, C. Briand, P.J. Derrick, F. Barras, Repair of oxidized proteins. Identification of a new methionine sulfoxide reductase, J. Biol. Chem. 276 (2001) 48915-48920.

[61] O. Yermolaieva, R. Xu, C. Schinstock, N. Brot, H. Weissbach, S.H Heinemann, T. Hoshi, Methionine sulfoxide reductase A protects neuronal cells against brief hypoxia/reoxygenation, Proc. Natl. Acad. Sci. U. S. A. 101 (2004) 1159-1164.

[62] J.E. Whittier, X. Yiong, M.C. Rechsteiner, T.C. Squier, Hsp90 enhances degradation of oxidized calmodulin by the $20 \mathrm{~S}$ proteasome, J. Biol. Chem. 279 (2004) (Electronic Publication M406048200)

[63] J.S. Beckman, W.H. Koppenol, Nitric oxide, superoxide, and peroxynitrite: the good, the bad, and ugly, Am. J. Physiol. 271 (1996) C1424-C1452.

[64] R. Radi, Nitric oxide, oxidants, and protein tyrosine nitration, Proc. Natl. Acad. Sci. U. S. A. 101 (2004) 4003-4008.

[65] S.S. Marla, J. Lee, J.T. Groves, Peroxynitrite rapidly permeates phospholipid membranes, Proc. Natl. Acad. Sci. U. S. A. 94 (1997) $14243-14248$.
[66] C.D. Reiter, R.-J. Teng, J.S. Beckman, Superoxide reacts with nitric oxide to nitrate tyrosine at physiological $\mathrm{pH}$ via peroxynitrite, J. Biol. Chem. 275 (2000) 32460-32466.

[67] S.V. Lymar, J.K. Hurst, Carbon dioxide: physiological catalyst for peroxynitrite-mediated cellular damage or cellular protectant? Chem. Res. Toxicol. 9 (1996) 845-850.

[68] M. Tien, B.S. Berlett, R.L. Levine, P.B. Chock, E.R. Stadtman, Peroxynitrite-mediated modification of proteins at physiological carbon dioxide concentrations: $\mathrm{pH}$ dependence of carbonyl formation, tyrosine nitration, and methionine oxidation, Proc. Natl. Acad. Sci. U. S. A. 96 (1999) 7809-7814.

[69] Y.-Z. Ye, M. Strong, Z.-Q. Huang, J.S. Beckman, Antibodies that recognize nitrotyrosine, Methods Enzymol. 269 (1996) 201-209.

[70] M-L. Brennan, W. Wu, Z. Shen, W. Song, H. Frost, C. Vadseth, L. Narine, E. Lenkiewicz, M.T. Borchers, A.J. Lusis, J.J. Lee, N.A. Lee, H.M. Abu-Soud, H. Ischiropoulos, S.L. Hazen, A tale of two controversies: defining both the role of peroxidases in nitrotyrosine formation in vivo using eosinophil peroxidase and myeloperoxidase-deficient mice, and the nature of peroxidasegenerated reactive nitrogen species, J. Biol. Chem. 277 (2002) $17415-17427$.

[71] M.B. Reid, W.J. Durham, Generation of reactive oxygen and nitrogen species in contracting skeletal muscle: potential impact on aging, Ann. N.Y. Acad. Sci. 959 (2002) 108-116.

[72] L. Kobzik, M.B. Reid, D.S. Bredt, J.S. Stamler, Nitric oxide in skeletal muscle, Nature 372 (1994) 546-548.

[73] L. Kobzik, B. Stringer, J.L. Balligand, M.B. Reid, J.S. Stamler, Endothelial type nitric oxide synthase in skeletal muscle fibers: mitochondrial relationships, Biochem. Biophys. Res. Commun. 211 (1995) 375-381.

[74] H. Bito, K. Deisseroth, R.W. Tsien, CREB phosphorylation and dephosphorylation: a $\mathrm{Ca}(2+)$ - and stimulus duration-dependent switch for hippocampal gene expression, Cell 87 (1996) 1203-1214.

[75] E.N. Olson, R.S. Williams, Remodeling muscles with calcineurin, Bioessays 22 (2000) 510-519.

[76] R. Bassel-Duby, E.N. Olson, Role of calcineurin in striated muscle: development, adaptation, and disease, Biochem. Biophys. Res. Commun. 311 (2003) 1133-1141.

[77] Y. Hitomi, T. Kizaki, T. Katsumura, M. Mizuno, C.E. Itoh, K. Esaki, Y. Fujioka, T. Takemasa, S. Haga, H. Ohno, Effect of moderate acute exercise on expression of mRNA involved in the calcineurin signaling pathway in human skeletal muscle, IUBMB Life 55 (2003) 409-413.

[78] T. Zhang, S. Miyamoto, J.H. Brown, Cardiomyocyte calcium and calcium/calmodulin-dependent protein kinase II: friends or foes? Recent Prog. Horm. Res. 59 (2004) 141-168.

[79] E. Carmeli, R. Coleman, A.Z. Reznick, The biochemistry of aging muscle, Exp. Gerontol. 37 (2002) 477-489.

[80] A.M. Janczewski, H.A. Spurgeon, E.G. Lakatta, Action potential prolongation in cardiac myocytes of old rats is an adaptation to sustain youthful intracellular $\mathrm{Ca}^{2+}$ regulation, J. Mol. Cell. Cardiol. 34 (2002) 641-648.

[81] E. Gutman, V. Hanzilikova, F. Vyskocil, Age changes in cross striated muscle of the rat, J. Physiol. 219 (1971) 331-343.

[82] L. Larsson, L. Edstrom, Effects of age on enzyme-histochemical fibre spectra and contractile properties of fast- and slow-twitch skeletal muscle in the rat, J. Neurol. Sci. 76 (1986) 69-89.

[83] N. Narayanan, D.L. Jones, A. Xu, J.C. Yu, Effects of aging on sarcoplasmic reticulum function and contraction duration in skeletal muscle of the rat, Am. J. Physiol. 271 (1996) C1032-C1040 (Cell Physiol 40).

[84] Y. Hayashi, Y. Sawa, W.S. Ohtake, N. Fukuyama, H. Nakazawa, H. Matsuda, Peroxynitrite detected in human myocardium after ischemia reperfusion, Ann. Thorac. Surg. 72 (2001) 571-576.

[85] A.J. Lokuta, N.A. Maertz, J.F. Kamp, H.H. Valdivia, R.A. Haworth, Increased tyrosine nitration of SERCA2a in human heart failure inhibits SR Ca-pump function, Biophys. J. 82 (2002) 597a. 
[86] T. Adachi, R. Matsui, S. Xu, M. Kirber, H.L. Lazar, V.S. Sharov, Ch. Schoneich, R.A. Cohen, Antioxidant improves smooth muscle arco/endoplasmic reticulum $\mathrm{Ca}(2+)$-ATPase function and lowers tyrosine nitration in hypercholesterolemia and improves nitric oxide-induced relaxation, Circ. Res. 90 (2002) 1114-1121.

[87] C.R. Ruse, F.-L. Tan, M. Kinter, M. Bond, Integrated analysis of the human cardiac transcriptome, proteome and phosphoproteome, Proteomics 4 (2004) 1505-1516.

[88] C. Toyoshima, H. Nomura, Structural changes in the calcium pump accompanying the dissociation of calcium, Nature 418 (2002) 605-611.

[89] R.I. Viner, A.F.R. Hühmer, D.J. Bigelow, Ch. Schöneich, The oxidative inactivation of sarcoplasmic reticulum $\mathrm{Ca}^{2+}$-ATPase by peroxynitrite, Free Radic. Res. 24 (1996) 243-259.

[90] C.J. Brandl, N.M. Green, B. Korczak, D.H. MacLennan, Two Ca ${ }^{2+}$ ATPase genes: homologies and mechanistic implications of deduced amino acid sequences, Cell 44 (1986) 597-607.

[91] D.A. Ferrington, Q. Yao, T.C. Squier, D.J. Bigelow, Comparable levels of Ca-ATPase inhibition by phospholamban in slow-twitch skeletal and cardiac sarcoplasmic reticulum, Biochemistry 41 (2002) $13280-13296$.

[92] S. Negash, L.T. Chen, D.J. Bigelow, T.C. Squier, Phosphorylation of phospholamban by cAMP-dependent protein kinase enhances interactions between Ca-ATPase polypeptide chains in cardiac sarcoplasmic reticulum membranes 35 (1996) 11247-11259.

[93] S. Negash, S. Huang, T.C. Squier, Rearrangement of domain elements of the Ca-ATPase in cardiac sarcoplasmic reticulum membranes upon phospholamban phosphorylation, Biochemistry 38 (1999) 8150-8158.

[94] S.A. Tatulian, B. Chen, J. Li, S. Negash, C.R. Middaugh, D.J. Bigelow, T.C. Squier, The inhibitory action of phospholamban involves stabilization of alpha-helices within the Ca-ATPase, Biochemistry 41 (2002) 741-751.

[95] B. Alvarez, G. Ferrer-Sueta, B.A. Freeman, R. Radi, Kinetics of peroxynitrite reaction with amino acids and human serum albumin, J. Biol. Chem. 274 (1999) 842-848.

[96] W.A. Jackson, J. Colyer, Translation of $\mathrm{Ser}^{16}$ and $\mathrm{Thr}^{17}$ phosphorylation of phospholamban into $\mathrm{Ca}^{2+}$-pump stimulation, Biochem. J. 316 (1996) 201-207.

[97] T. Cantilina, Y. Sagara, G. Inesi, L.R. Jones, Comparative studies of cardiac and skeletal sarcoplasmic reticulum ATPases. effect of a phospholamban antibody on enzyme activation by $\mathrm{Ca}^{2+}$, J. Biol. Chem. 268 (1993) 17018-17025.

[98] C. Toyoshima, G. Inesi, Structural basis of ion pumping by $\mathrm{Ca}^{2+}$ ATPase of the sarcoplasmic reticulum, Annu. Rev. Biochem. 73 (2004) 269-292.

[99] S. Huang, T.C. Squier, Enhanced rotational dynamics of the phosphorylation domain of the Ca-ATPase upon calcium activation, Biochemistry 37 (1998) 18064-18073.

[100] C. Toyoshima, M. Nakasako, H. Nomura, H. Ogawa, Crystal structure of the calcium pump of sarcoplasmic reticulum at $2.6 \mathrm{~A}$ resolution, Nature 405 (2000) 647-655.

[101] C. Toyoshima, T. Mitzutani, Crystal structure of the calcium pump with a bound ATP analogue, Nature 430 (2004) 529-535.
[102] B. Chen, T.C. Squier, D.J. Bigelow, Calcium activation of the CaATPase enhances conformational heterogeneity between nucleotide binding and phosphorylation domains, Biochemistry 43 (2004) 4366-4374.

[103] Y. Kimura, K. Kurzydlowsi, M. Tada, D.H. MacLennan, Phospholamban regulates the $\mathrm{Ca}^{2+}$-ATPase through intramembrane interactions, J. Biol. Chem. 271 (1996) 21726-21731.

[104] T. Toyofuku, K. Kurzydlowski, M. Tada, D.H. MacLennan, Amino acids Glu2 to Ile18 in the cytoplasmic domain of phospholamban are essential for functional association with the $\mathrm{Ca}^{2+}$-ATPase of sarcoplasmic reticulum, J. Biol. Chem. 269 (1994) 3088-3094.

[105] T. Toyofuku, K. Kurzydlowski, M. Tada, D.H. MacLennan, Amino acids Lys-Asp-Asp-Lys-Pro-Val402 in the $\mathrm{Ca}^{2+}$-ATPase of cardiac sarcoplasmic reticulum are critical for functional association with phospholamban, J. Biol. Chem. 269 (1994) 22929-22932.

[106] S. Negash, Q. Yao, H. Sun, J. Li, D.J. Bigelow, T.C. Squier, Phospholamban remains associated with the $\mathrm{Ca}^{2+}$ and $\mathrm{Mg}^{2+}$ dependent ATPase following phosphorylation by cAMP-dependent protein kinase, Biochem J. 351 (2000) 195-205.

[107] M. Asahi, N.M. Green, K. Kurzydlowsi, M. Tada, D.H. MacLennan, Phospholamban domain IB forms an interaction site with the loop between transmembrane helices M6 and M7 of sarco(endo)plasmic reticulum $\mathrm{Ca}^{2+}$-ATPases, Proc. Natl. Acad. Sci. U. S. A. 98 (2001) $10061-10066$.

[108] B. Chen, D.J. Bigelow, Phosphorylation induces a conformational transition near the lipid-water interface of phospholamban reconstituted with the Ca-ATPase, Biochemistry 41 (2002) $13965-13972$.

[109] J. Li, D.J. Bigelow, T.C. Squier, Conformational changes within the cytosolic portion of phospholamban upon release of Ca-ATPase inhibition, Biochemistry 43 (2004) 3870-3879.

[110] J. Li, D.J. Bigelow, T.C. Squier, Phosphorylation by cAMPdependent protein kinase modulates the structural coupling between the transmembrane and cytosolic domains of phospholamban, Biochemistry 42 (2003) 10674-10682.

[111] J. Li, Y. Xiong, D.J. Bigelow, T.C. Squier, Phospholamban binds in a compact and ordered conformation to the Ca-ATPase, Biochemistry 43 (2004) 455-463.

[112] J.S. Richardson, D.C. Richardson, Principles and patterns in protein conformation, in: G.D. Fasman (Ed.), Prediction of Protein Structure and the Principles of Protein Conformation, Plenum Press, New York, pp. 1-98.

[113] M.O. Stojanovic, M.T. Ziolo, G.M. Wahler, B.M. Wolska, Antiadrenergic effects of nitric oxide donor SIN-1 in rat cardiac myocytes, Am. J. Physiol., Cell Physiol. 281 (2001) C342-C349.

[114] S. Lamberth, H. Schmid, M. Muenchbach, T. Vorherr, J. Krebs, E. Carafoli, C. Griesinger, NMR solution structure of phospholamban, Helv. Chim. Acta 83 (2000) 2141-2152.

[115] R.A. Sayle, E.J. Milner-White, RASMOL: biomolecular graphics for all, Trends Biochem. Sci. 20 (1995) 374-376.

[116] Y. Yao, T.C. Squier, Variable conformation and dynamics of calmodulin complexed with peptides derived from the autoinhibitory domains of target proteins, Biochemistry 35 (1996) 6815-6827. 